\title{
La decisión de estudiar el doctorado en México o en el extranjero: ¿determinación social, herencia de rutas académicas o construcción de destinos?
}

\author{
Mónica López Ramírez
}

\section{Resumen}

La investigación de la cual se desprende la presente nota de investigación busca comprender la manera en la cual estudiantes mexicanos de ingeniería elijen destinos de formación doctoral (nacionales o extranjeros). Con ello, se intenta profundizar en dos procesos que confluyen en este tipo de decisiones: las transiciones educativas en este nivel de estudios y la salida del país con fines académicos.

Lo anterior implica uno de los principales desafíos de la investigación: el desarrollo de un modelo analítico que permita vincular ambos procesos, a la vez que se dé cuenta de los elementos y actores que participan en la decisión y las formas en que éstos se relacionan. El propósito del presente trabajo es mostrar los elementos que conforman este modelo, dilucidar su construcción y los supuestos sobre los que subyace.

Palabras clave: movilidad internacional, toma de decisión, estudiantes de posgrado. 


\section{Abstract}

\section{The decision to study a PhD in Mexico or abroad: social determination, academic heritage routes or construction of destinations?}

The research from which is derived the present research note aims to understand the way in which Mexican engineering students choose destinations of doctoral training (national or abroad). Thereby, I try to study two processes that converge in this decision: educational transitions in this level of education and leaving the country for academic purposes. This implies one of the main research challenges: developing an analytical model to link the two processes, and at the same time, realize the elements and actors involved in the decision and the ways in which they relate. The purpose of this paper is to shows the elements of this model, elucidating its construction and the assumptions underlying.

Key words: international mobility, decision making, postgraduae students.

\section{Introducción}

La movilidad internacional para realizar estudios ha sido una realidad para muchos países, incluido México, cuyos estudiantes constituyen el conjunto más numeroso de universitarios latinoamericanos en el exterior (Luchilo, 2008). Este tipo de movilidad cobra relevancia por el aumento constante que registra año con año, con características disímiles en cuanto a patrones de destino, participación por campo de conocimiento y fuentes de financiamiento.

El tema ha sido abordado desde la década de los cincuenta respondiendo a los cambios en la percepción social y cultural, y presentando dificultades conceptuales derivadas de la heterogeneidad de situaciones comprendidas en el concepto. Las diversas investigaciones se han centrado en distintas aristas del fenómeno, por lo que aún son escasos los trabajos sobre los principales actores de la movilidad internacional y, en menor medida, aquellos relacionados con el análisis del proceso de toma de decisión de los estudiantes sobre permanecer en sus países de origen o salir para continuar su formación académica.

La investigación de la cual se desprende la presente nota de investigación busca comprender la manera en la cual estudiantes mexicanos de ingeniería elijen destinos de formación doctoral (nacionales o extranjeros). Con ello, se intenta profundizar en dos procesos que confluyen en este tipo de decisiones: las transiciones educativas en este nivel de estudios y la salida del país con fines académicos. 
Lo anterior implica uno de los principales desafíos de la investigación, el desarrollo de un modelo analítico que permita vincular ambos procesos, a la vez que se dé cuenta de los elementos y actores que participan en la decisión y las formas en que éstos se relacionan.

Para ello, el trabajo parte de consideraciones contextuales que dan cuenta del problema en cuestión, posteriormente se presentan los principales lineamientos teóricos en que sustenta la investigación y que muestran la construcción de un modelo específico; por último, se plantea la estrategia metodológica.

\section{El problema de investigación}

La movilidad internacional de estudiantes representa la faceta más notoria de la internacionalización de la educación superior. En la última década (2000-2010) el número de estudiantes que se encontraban fuera de sus países de origen desarrollando estudios se duplicó alcanzando los 3.8 millones (UNESCO, 2013). Estos estudiantes móviles salen principalmente de China (17.1\%), India (5.2\%) y Corea (3.4\%), para dirigirse a Estados Unidos (19\%), Reino Unido (11\%), Francia (7\%), Alemania (5\%) y Japón (4\%).

En el caso específico de México, por cada cinco personas con maestría y tres con doctorado en el país, se encuentra una en Estados Unidos (Tuirán, 2009), lo cual refleja la existencia de una dinámica de formación en el exterior para algunos estudiantes mexicanos.

Se prevé que estas tendencias se incrementen dada la proliferación de programas que promueven la movilidad de estudiantes, la facilidad de ingreso y atractivo de algunas opciones foráneas frente a las locales y por el prestigio que pueden ostentar los títulos obtenidos en el extranjero en el mercado laboral cada vez más globalizado.

En México este tipo de movilidad está estrechamente ligada a la evolución del posgrado nacional y al desarrollo y tendencias en los apoyos económicos, principalmente gubernamentales. En ambos casos, el Consejo Nacional de Ciencia y Tecnología (Conacyt) representa un actor fundamental, ya que es el principal instrumento con que cuenta el Estado para orientar sistemáticamente y a gran escala la formación de recursos de alto nivel a través del programa de becas. Este programa otorga ayuda económica para realizar estudios de posgrado en sus diferentes niveles dentro del territorio mexicano y fuera de él.

A lo largo del desarrollo del programa y del fortalecimiento y consolidación del posgrado nacional, el apoyo gubernamental ha presentado 
cambios significativos, entre los que destacan: a) la canalización de apoyos a programas nacionales frente a internacionales como una medida para fortalecer los programas de posgrado locales; $b$ ) la prioridad en la asignación de las becas al extranjero en áreas estratégicas para el desarrollo nacional, principalmente aquellas relacionadas con ingeniería y ciencia aplicada; c) la canalización de recursos, sobre todo extranjeros, hacia el nivel de doctorado y; d) la diversificación hacia destinos específicos (Estados Unidos, Inglaterra, Francia, España, Canadá y Alemania), los cuales concentran $80 \%$ de becarios mexicanos en el exterior.

Estos destinos concuerdan con datos sobre la formación de académicos mexicanos en el extranjero (Didou, 2010; Gérard y Grediaga, 2009), por lo que se menciona que algunos países representan polos de atracción de estudiantes en función de los saberes impartidos y su legitimidad (Gérard y Maldonado, 2009).

La movilidad por estudios no obedece siempre, o prioritariamente, a una cercanía geográfica, aun cuando todo indica que la segunda favorece la primera; pareciera ser resultado de las dinámicas científicas institucionales entre México y los países de destino de los estudiantes (Didou y Gérard, 2011), de las relaciones y vínculos establecidos como producto de tradiciones científicas y disciplinarias de formación en destinos específicos y de las políticas públicas nacionales de apoyo e internacionales para atraer estudiantes (Grediaga y Maldonado, 2012; Gérard, 2014).

En este contexto, la movilidad también es resultado de las expectativas e intereses particulares de los individuos para continuar su formación y desarrollar las habilidades y conocimientos necesarios que les permitan incorporarse en el mercado laboral, académico o científico. Pero, ¿quiénes deciden permanecer en el país para continuar su formación? ¿Qué los lleva a optar por ello? ¿Qué factores intervienen en la decisión? ¿Cómo es el proceso de decidir continuar la formación académica en México o en el extranjero?

En este último punto se sitúa esta investigación. Desde la óptica de los estudiantes, decidir permanecer en México o salir para continuar su formación doctoral implica, por una parte, transitar hacia el último nivel en su trayectoria formativa y, por otra, una movilidad geográfica fuera del país. Por su naturaleza, esta decisión conlleva la definición de un proyecto de vida que reordena y pone en juego diversos elementos con profundas implicaciones en el ámbito emocional, familiar, personal, académico y laboral.

Esta decisión incluye, además, el desafío particular de considerar un cambio de entorno, la adaptación e independencia que ello conlleva, una constante incertidumbre sobre los destinos educativos y geográficos y sobre los procesos inmersos en la transición educativa hacia este nivel, así como 
un incesante auto cuestionamiento sobre las capacidades y habilidades personales y académicas, y sobre los riesgos y beneficios que implica la decisión. Por otro lado, representa también la curiosidad, emoción y adrenalina que envuelve a lo nuevo y desconocido.

En ese sentido, la investigación busca comprender la manera en la cual estudiantes mexicanos de ingeniería elijen destinos de formación doctoral, a través del análisis de la interacción entre la agencia que los estudiantes ejercen a partir de sus propias circunstancias y motivaciones construidas a lo largo de su historia de vida, y de los recursos y límites de los contextos en los que se desarrollan.

\section{Antecedentes y lineamientos teóricos}

La salida de científicos y personal calificado de sus países de origen ha estado envuelta en un constante debate sobre los beneficios y perjuicios que esto representa, tanto para los países de salida, como para los de arribo. A lo largo del tiempo se han utilizado e intercambiado diversos términos para intentar dar un cambio en las aproximaciones conceptuales y en la percepción social y cultural del fenómeno.

Lo anterior solo ha acentuado la confusión sobre lo que se entiende por movilidad internacional, la discrepancia respecto de a quiénes se hace alusión cuando se habla del tema, y a entenderla como un fenómeno estático.

La investigación en curso parte de tres precisiones fundamentales: en primer lugar, que la movilidad internacional se refiere al proceso de cursar estudios en una institución de educación superior de otro país distinto al de origen en un determinado tiempo (Altbach, 1998).

En segundo lugar, que la población a la cual se hará mención al hablar de movilidad internacional serán los estudiantes de posgrado; y en tercer lugar, que se entenderá la movilidad como un proceso conformado por diversas etapas: la decisión de viajar, la llegada al país extranjero y la decisión de retorno o no al país de origen (González Barea, 2008); del cual, interesa profundizar en el momento en el que se constituye la decisión de desarrollar estudios en un lugar diferente al de origen.

Con base en estas precisiones es que se establece que la movilidad internacional para realizar estudios conjuga una decisión que los individuos toman para continuar su trayectoria educativa, así como una decisión que implica movilidad geográfica de carácter internacional.

¿Cómo analizar esta amalgama de procesos? Al respecto, un cúmulo importante de trabajos intenta dar cuenta de ambas decisiones. 
En cuanto a las transiciones y decisiones educativas, el debate principal se mantiene entre explicaciones para las cuales las decisiones educativas pueden entenderse como los condicionamientos estructurales que se plasman en el desempeño diferencial de los estudiantes de distintos sectores sociales. La escuela es vista por estas posturas como la institución que legitima estas diferencias, en principio arbitrarias, por medio del sistema educativo a través de evaluaciones y exámenes basados en el mérito, así como por las disposiciones subjetivas que producen e interiorizan la subordinación (Bourdieu y Passeron, 1996 [1977]; Bernstain, 1990).

Estos postulados constituyen la base de una amplia investigación empírica para los que la continuación y selección de instituciones educativas está ligada con el origen social en diferentes situaciones: en la percepción sobre las instituciones y los recursos materiales y económicos asociados a la elección (Davey, 2012; Ball et al., 2002), en relación con el capital social con el que cuentan los estudiantes y sus familias (Greenbank, 2009) y respecto de las características y recursos de las instituciones previas a la toma de decisión a las que asisten los estudiantes (Ball et al., 2002; Reay, David y Ball, 2001).

Por otra parte, otro conjunto de explicaciones postulan que las decisiones sobre la continuación escolar dependen de procesos racionales de decisión con arreglo a fines, en donde los recursos económicos y el rendimiento escolar son los elementos a considerar para dicho cálculo. Los efectos primarios (o de rendimiento) expresan la asociación entre el origen social y las opciones educativas de los individuos mediada por el rendimiento cognitivo y académico, mientras que los efectos secundarios (o de elección) son generados por las diferencias socialmente estructuradas en la sensibilidad y las percepciones acerca de los costos y beneficios de las inversiones educativas, así como en los riesgos percibidos asociados con la probabilidad esperada de éxito de los estudiantes (Boudon, 1973; Breen y Goldthorpe, 1997).

Las investigaciones empíricas al respecto establecen que las desigualdades de acceso a ciertas opciones estratificadas en las primeras etapas de la trayectoria educativa se trasladan a la participación en la universidad (Contini y Scagni, 2013; Neugebauer et al., 2013), que en la transiciones de nivel superior los efectos secundarios recobran importancia (Büchner y Van del Velden, 2013) y que las desigualdades de orígenes sociales siguen siendo evidentes en el paso hacia la universidad (Jackson, 2013).

Desde ambas perspectivas, el papel del origen social es preponderante al hacer inteligibles para los estudiantes opciones como posibles, deseables y apropiadas en relación con sus posiciones de clase. Desde el plano racional, son la base desde las cuales se hacen estimaciones de costo y probabilidades de éxito y beneficio; mientras que el enfoque reproduccionista, se trata de 
estructuras en las que los individuos pueden ejercitar sus elecciones a través del habitus de clase.

Sin embargo, estos esquemas de explicación presentan diversas restricciones para analizar la decisión de interés: desde estos enfoques existe una predeterminación de destinos escolares según el origen social de pertenencia, en donde es visible la ausencia de la agencia de los individuos. Aunado a ello, el tratamiento que se le da a las decisiones es estático, analizado en un punto determinado sin considerar elementos temporales ligados a la decisión $y$, en el caso del enfoque racional, no se consideran las imperfecciones de la racionalidad. ${ }^{1}$

Las explicaciones sobre decisiones educativas aportan elementos importantes aunque no suficientes para dar cuenta de la decisión de interés para la investigación.

¿Qué elementos para el análisis brindan las investigaciones sobre la decisión de continuar los estudios en el extranjero?

Los trabajos al respecto se agrupan en dos vetas de investigaciones: aquellas que dan explicaciones a partir de las características de los contextos de salida y recepción en los cuales se encuentran los estudiantes como preponderantes en la decisión, y otro grupo de trabajos para el cual la salida es resultado de la reproducción de desigualdades sociales o de rutas académicas de formación.

El primer grupo, reunido en torno a la perspectiva del push-pull, privilegia explicaciones a partir de factores que empujan a los estudiantes de sus países de origen (como factores institucionales, económicos, políticos) y aquellos que atraen a los estudiantes hacia el extranjero (el prestigio de los títulos obtenidos, el grado de selectividad de las instituciones y el atractivo de la vida y cultura que ostentan otros países) (Altbach, 2004; Van Mol, 2008, Chen, 2007; Cubillo, Sánchez y Cerviño, 2006).

Chen (2007), Galotti y coatores (2006), así como Bogdan, Bartkowiak y Skuza (2012), incorporan en esta perspectiva la idea de que estos factores influyen en diferentes momentos: en un primer momento de predisposición las características como el origen social, preferencias y habilidades académicas de los estudiantes ejercerán mayor influencia, por lo que los consideran factores de empuje. En un segundo momento de búsqueda de información sobre las opciones, allegarse a fuentes de información y su uso será lo preponderante.

${ }^{1}$ Dado que para decidir racionalmente se debe contar con toda la información para evaluar las alternativas, en la realidad la racionalidad es imperfecta, ya que no se puede tener en todos los casos la totalidad de información para tomar una decisión racional, ni tampoco es posible una "maximización global" (Cristiano, 2006). En ese sentido, al no conocer todas las opciones posibles los sujetos no decidirían con base en maximizar sus ganancias, sino en minimizar sus riesgos. 
En un tercer momento de evaluación, la valoración sobre las opciones en el extranjero, trámites y ayudas financieras, representaran factores de jale positivos y negativos.

Por otra parte, el segundo grupo de trabajo privilegia el papel del origen social como determinante no sólo del ingreso a la educación en el nivel de posgrado, sino a los destinos y trayectorias de los individuos en ellos. Se trata de una estrategia mediante la cual las clases buscan reproducirse y conservar su ventaja social (Waters y Brooks, 2010).

Para estas investigaciones, los estudiantes móviles pertenecen a una élite migratoria procedentes de los grupos sociales más altos y con padres con mayores logros educativos; estos estudiantes traen consigo un background de viaje expresado en variables como el dominio de otros idiomas, experiencias previas de movilidad desarrolladas en el ambiente familiar o cierta cultura viajera, que les permite optar por desarrollar una experiencia académica en el extranjero. Estas características permean también la propia configuración de que la opción de estudiar fuera del país es plausible (Waters y Brooks, 2010; West et al., 2000; González Barea, 2008; Salisbury et al., 2009; Luchilo, 2008).

Desde esta perspectiva, la movilidad de estudiantes está estructurada por fuerzas más allá de las opciones o preferencias individuales y está restringida a las clases trabajadoras que tienen menos oportunidad de participar debido a sus limitaciones financieras y lingüísticas propias de los ambientes y la cultura socioeconómica en los que están inmersos (Bernstein, 1985).

El último grupo de trabajos parte de la misma idea de que la movilidad internacional es resultado de un proceso de reproducción, pero en este caso no se trata de orígenes sociales, sino de una reproducción de destinos educativos. En ese sentido, este grupo de investigaciones considera que a través de las relaciones o lazos entre académicos en diversos países, que constituyen cadenas de saber (Gérard y Maldonado, 2009), se despierta el deseo de los estudiantes de formarse en el extranjero, asociado a la idea de una manera específica de hacer ciencia, una escuela de pensamiento o una figura tutelar (Didou, 2005; Van Der Meid, 2003).

Las cadenas de saber generan modelos transgeneracionales de formación en ciertas disciplinas (Gérard y Grediaga, 2009), a la vez que actúan como reproductores de un ethos y una manera de hacer ciencia específica mediante la socialización de sus cuadros. Desde estas posturas no se discurre sobre las estrategias individuales para acceder a estas cadenas de saber que a su vez pueden estar influidas por el origen social, ni tampoco sobre la conformación de trayectorias de los estudiantes por estar integrados a ellas.

La revisión de estas investigaciones permite considerar, por un lado, la noción procesual de la toma de decisión comprendida por diversas etapas en 
la cual intervienen elementos específicos en cada una de ellas; y por otro, refuerza la idea de incluir el papel de los contextos institucionales como espacios de conformación de redes, reproducción de saberes y lazos para establecer conexiones con instancias internacionales que pueden constituir elementos centrales en el desarrollo de la trayectoria de los jóvenes científicos (Didou y Gérard, 2011).

Debe destacarse que los hallazgos de estas investigaciones respecto de la idea de habilidades y disposiciones hacia la extranjero (Waters y Brooks, 2010; Luchilo, 2008) y del papel del dominio de un segundo idioma, como canal para la movilidad cuando se tiene cierta habilidad o como un poderoso obstáculo al carecer de ella (Mei y Bray, 2007; González Barea, 2008; Findlay et al., 2006), brindan la pauta para prestar mayor atención a estos elementos.

Por ello, en la investigación se propone integrar este conjunto de atributos en un tipo específico de capital, al que se ha denominado capital viajero.

Esta propuesta conceptual permite ir más allá de la idea de una cultura viajera o de comunicación o habilidades interculturales (Spitzberg, 1997; Rehaag, 2008). Se trata de un punto de confluencia de diversos capitales: de capital cultural, principalmente institucionalizado, a través de la inversión personal en la inculcación y asimilación de hábitos, disposiciones y competencias como el aprendizaje de idiomas y del contacto con otras culturas a través de los viajes por cuestiones turísticas o por haber residido en el extranjero en algún momento. Ello a su vez implica contar con el capital económico necesario para movilizarse fuera del país, o bien, con una suerte de capital social que permita mantener contacto con personas (principalmente familiares) que se encuentren en el extranjero.

Este tipo de capital implicaría una serie de disposiciones y actitudes reflejadas en la comodidad de negociación de entornos multiculturales, la conciencia y el interés en lo internacional, cierta apertura a la diversidad, al desafío y al reto que implica "lo extranjero", y hasta cierto punto se podría hablar de certidumbre en las habilidades de conducción y adaptación en diversos contextos.

Todo ello adquirido antes de la toma de decisión, principalmente, al interior de los contextos familiares, por lo que se trataría de una forma de representación de las diferencias de origen social que siguen teniendo preponderancia en las decisiones de los estudiantes con cierto grado de madurez e independencia familiar, ya que estas habilidades se suponen necesarias al momento de decidir permanecer o salir del país con fines académicos.

Para cerrar esta sección, basta decir que cada una de las posturas revisadas, desde la decisión sobre la cual se enfocan, aporta elementos que 
abonan a conforma un modelo de análisis sobre el problema específico de esta investigación; sin embargo, si se optara por adherirse a los preceptos de alguna de ellas en particular, asumiríamos una perspectiva parcial para explicar la decisión de interés.

Además de estas posturas, deben considerarse también las limitaciones de las cuales adolecen; en específico, el que consideran la toma de decisión como algo estático, por lo que es necesario adoptar una perspectiva que permita introducir el factor temporal en el proceso de toma de decisión, a la vez que se consideran elementos de dimensiones micro y macro sociales.

En este sentido la perspectiva del curso de vida permite analizar la implicación de los individuos en distintos dominios institucionales a lo largo de la vida, haciendo énfasis en los eventos (la entrada al mercado de trabajo, compromisos matrimoniales, tener hijos, la salida del hogar parental), que marcan cambios importantes en esta implicación o en la forma en que estos eventos se entrelazan unos con otros para conformar trayectorias específicas (Elder, 1992), a la par que permite entender las trayectorias individuales en un marco de condiciones históricas específicas que les imponen restricciones. $^{2}$

Con ello se pretende establecer un modelo analítico que agrupe diversos elementos para dar cuenta de una decisión compleja.

\section{EI modelo analítico}

Lo anterior no hace más que resaltar la necesidad de un enfoque integrado que pueda contemplar esa complejidad en el abordaje. Es clara la necesidad de plantear el fenómeno desde una perspectiva analítica que sea capaz de entenderlo como efectos combinados entre la conducta individual, la articulación colectiva y factores estructurales de acotación.

En ese sentido, en la propuesta analítica desarrollada se considera que:

1. La decisión es un proceso dinámico y cambiante, que forma parte de decisiones previas sobre un estilo de vida específico. La decisión está enclavada en diferentes tipos de biografías, diversas estructuras de

${ }^{2}$ Asumir los principios de la perspectiva del curso de vida permite afrontar la restricción temporal y explorar otras dimensiones, específicamente, la concurrencia de eventos y variaciones de los orígenes sociales a lo largo de la vida de los estudiantes y en diferentes dominios institucionales que puedan otorgar significado a sus decisiones. 
Figura 1

Proceso de toma de decisión sobre el lugar para estudiar el doctorado

\begin{tabular}{|c|c|c|}
\hline $\begin{array}{c}\text { Etapa 1: } \\
\text { Predisposición }\end{array}$ & $\begin{array}{l}\text { Etapa 2: } \\
\text { Búsqueda }\end{array}$ & $\begin{array}{l}\text { Etapa 3: } \\
\text { Decisión }\end{array}$ \\
\hline $\begin{array}{c}\text { Continuar } \\
\text { doctorado } \\
\text { Maestría- } \\
\text { Licenciatura }\end{array}$ & $\begin{array}{l}\text { Búsqueda de } \\
\text { información } \\
\text { y solicitud } \\
\text { a programas } \\
\text { de posgrado } \\
\text { y de apoyos } \\
\text { económicos }\end{array}$ & $\begin{array}{l}\text { Doctorado } \\
\text { en } \\
\text { México }\end{array}$ \\
\hline No continuar & & \\
\hline
\end{tabular}

Fuente: elaboración propia.

oportunidades institucionales, de relaciones sociales (Ball et al., 2002) y horizontes de acción segmentados (Hodkinson y Sparkes, 1997; Heinz, 2009).

2. Al ser considerada como un proceso, la toma de decisión se entiende como una secuencia de etapas interrelacionadas entre sí, que comprenden la predisposición, la búsqueda y la decisión (Figura 1).

3. El proceso de toma de decisión sobre el lugar para realizar los estudios de doctorado es un fenómeno multidimensional, por lo que se incorporan elementos de: las condiciones sociales iniciales, referido al origen familiar y social desde el cual los estudiantes construyen el panorama de sus vidas, de los contextos institucionales a través de las características y procesos desarrollados al interior de las instituciones educativas previas a la toma de decisión, de los eventos derivados del curso de vida de los individuos, de la agencia relacionada con la planificación futura y la selección entre vías alternativas en determinadas circunstancias, y de la evaluación o estimación de alternativas y selección de una determinada opción. 
Figura 2

Modelo analítico

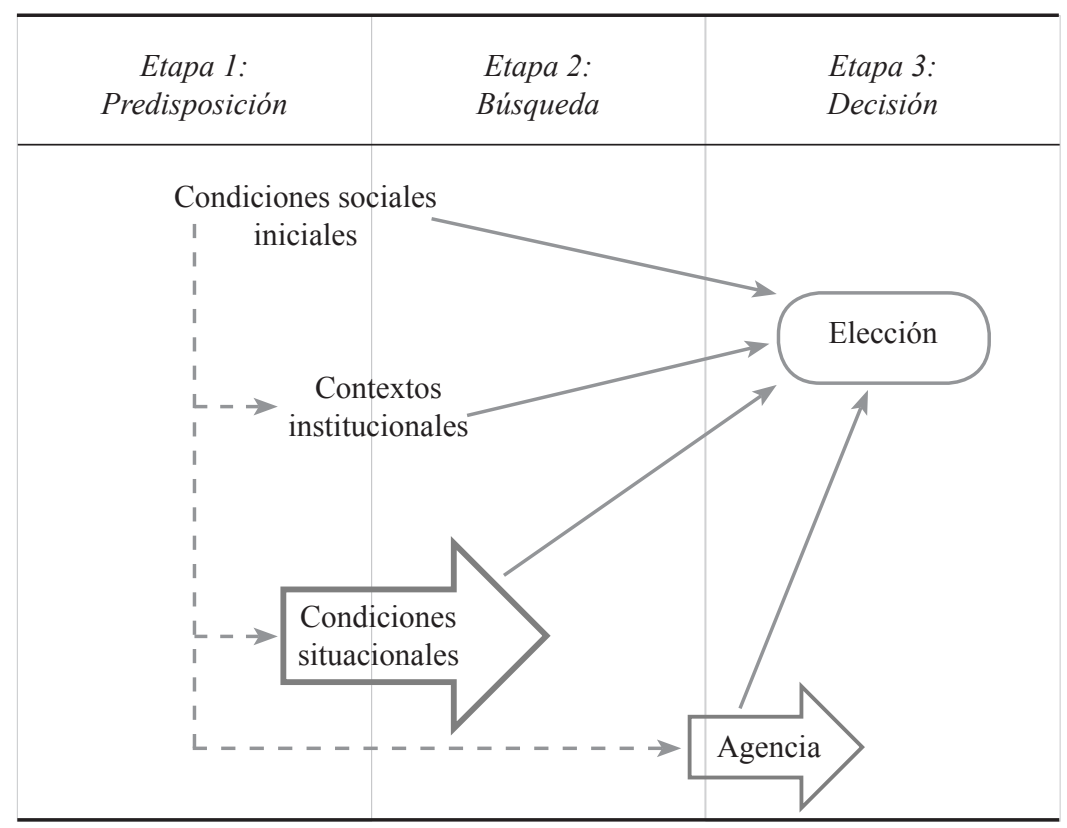

Con base en las consideraciones anteriores el modelo analítico establece la toma de decisión como un proceso conformado por diversas etapas interrelacionadas a través de las cuales se hacen latentes e interactúan un conjunto de elementos (Figura 2).

En este sentido, en la investigación se presupone que, en primer lugar, dado el hecho de que en las diversas transiciones y sus correspondientes decisiones educativas sigan imperando elementos ligados a la desigualdad social, hace suponer que en esta transición educativa persistan remanentes de la herencia social de los estudiantes; en segundo lugar, que los contextos educativos por los cuales transitan los estudiantes a lo largo de su trayectoria formativa pueden representar espacios de socialización y de adquisición de recursos que influyan en la decisión; finalmente, que estos elementos pueden interactuar de manera particular y establecer límites con diversa amplitud en los cuales los estudiantes actúen y elijan determinados destinos de formación. 


\section{Estrategia metodológica}

La estrategia metodológica que se lleva a cabo es de orden cualitativo. En efecto, a través del análisis de casos múltiples, sus observaciones y entrevistas con participantes, se muestra cómo se vive, articula y se transita en el terreno decisional.

Para ello, se decidió reconstruir el proceso de toma de decisión de estudiantes mexicanos en programas de doctorado nacionales y en el extranjero en el área de conocimiento de ingeniería y tecnología mediante entrevistas semiestructuradas. Lo anterior responde a la necesidad de comparar procesos de toma de decisión de estudiantes adscritos a IES y programas de posgrado con características estructurales divergentes y que han desarrollado en diferente medida, forma, tiempo y dinámicas, vínculos de colaboración con IES o programas de posgrado extranjeros específicos, lo que les brinda recursos de diversa índole a los cuales los estudiantes pueden o no acceder y que, se intuye, influyen en la decisión de los estudiantes.

A través de las trayectorias de vida y formativas de los estudiantes se permitirá dar cuenta del proceso de construcción y toma de decisión, entendido como una decisión que no se da en un momento específico, sino que se construye a través de la historia de vida del individuo, de las relaciones con otros y del acceso a recursos en determinados ámbitos educativos.

La elección de los ingenieros para el estudio responde a cuatro razones. En primer lugar, representa una de las áreas prioritarias ligadas al Plan Nacional de Desarrollo (PND) actual — desde 2003gubernamentales se canalizan a la formación de recursos humanos en este campo, orientados en mayor medida hacia el extranjero.

En segundo lugar, el aumento en el número de egresados en ingeniería en las dos últimas décadas coloca a México como una de las naciones que más ingenieros gradúa cada año, después de Estados Unidos, Japón y Corea (OCDE, 2010).

En tercer lugar, la inclinación por formarse como investigadores y científicos en esta área de conocimiento es menor con respecto a otras; a su vez, la inserción en el mercado industrial para los egresados en este campo presenta condiciones favorables con respecto a otras áreas. Finalmente, debido al desarrollo de la ingeniería en nuestro país, hoy día la formación de recursos en este campo se caracteriza por una alta heterogeneidad y estratificación.

Una vez definida el área de conocimiento, se seleccionaron tres programas de posgrado nacionales pertenecientes a las principales instituciones en el país con mayor tradición en la formación de ingenieros - IPN, UNAM e ITESM- A la vez que se eligieron tres de los principales países e institu- 
ciones de formación de ingenieros mexicanos en el extranjero (Estados Unidos-University of California, Francia-Institute National Polytechnique de Toulouse, Université Paul Sabatier, y Reino Unido-University of Manchester) para reconstruir y analizar comparativamente el proceso de toma de decisión por un destino formativo. ${ }^{3}$

Para el análisis planteado se realizaron 43 entrevistas con estudiantes mexicanos que desarrollan su doctorado en las instituciones mencionadas: 19 en programas de doctorado en México y 24 en posgrados en el extranjero. El trabajo de campo en México se realizó de agosto a diciembre de 2012; mientras que en el extranjero se hizo de enero a junio de 2013.

El análisis de las entrevistas ha privilegiado cuatro ejes:

a) la caracterización de los estudiantes de acuerdo con sus condiciones sociales iniciales (origen social, capital viajero y la trayectoria escolar previa),

b) se ha profundizado en las trayectorias educativas y laborales de los estudiantes, haciendo énfasis en los procesos de socialización formativa al interior de las instituciones educativas por las que han transitado para identificar elementos que intervienen en la decisión de los estudiantes entrevistados,

c) en las acciones, estrategias y agencia que los estudiantes despliegan en la búsqueda de información, evaluación y elección de distintas alternativas para continuar su formación doctoral y,

d) en la identificación de patrones de interacción existentes entre los elementos anteriormente referidos en el proceso de toma de decisión.

\section{A manera de conclusión}

La revisión realizada pone en evidencia el carácter complejo inmerso en el proceso de decidir continuar la formación doctoral en México o en el extranjero. El proceso comprende dos decisiones relacionadas: la transición hacia un nivel educativo y la movilidad geográfica fuera del país. Se entiende esta decisión como el resultado de la confluencia de elementos que se construyen

${ }^{3}$ Los casos fueron seleccionados previas entrevistas con actores clave: la actual Directora Adjunta del Programa de Becas, el ex director del mismo programa y personal de becas al extranjero de Conacyt; así como por el análisis de la base de datos de becarios Conacyt en el extranjero construida a partir de los datos proporcionados por dicha instancia, mediante el cual se pudo constatar que los países e instituciones seleccionados representan los principales destinos para los becarios mexicanos fuera del país, en general y para el caso de los ingenieros. 
a través de la historia de vida del individuo, de las relaciones con otros y del acceso a recursos en determinados ámbitos educativos.

El modelo que se presenta permite establecer un conjunto de factores que influyen en el proceso de toma de decisión con mayor presencia en determinadas etapas, así como la interrelación entre éstos. Además, reconoce la presencia de atributos y elementos que influyen, modifican y orientan diversos cursos de acción a lo largo de las diferentes etapas del proceso de toma de decisión.

En ese sentido la postura propuesta intenta fungir como herramienta de observación para entender el proceso desde el análisis de las experiencias de los actores, otorgando preponderancia a la experiencia individual y colectiva, y a las motivaciones construidas a lo largo del ciclo vital, sin restar importancia a recursos y límites más estructurales $\mathrm{y}$, sobre todo, a las — diferentes - interacciones entre ambos.

Recibido y revisado: enero de 2015

Correspondencia: Calle Hormiga núm. 61/Interior 5/Col. Tepeyac Insurgentes/correo electrónico: mlramirez@colmex.mx

\section{Bibliografía}

Altbach, Philip (2004), "Higher Education Crosses Borders", Change, núm. 36, marzo-abril, pp. 18-25.

Altbach, Philip (1998), "The Foreign Student Dilemma”, en P. Altbach (ed.), Comparative Higher Education. Knowledge, the University and Development, Hong Kong, Comparative Educational Research Centre-The University of Hong Kong, pp. 225-248.

Ball, Stephen, Jackie Davies, Miriam David y Diane Reay (2002), "Classification and Judgment: Social Class and the Cognitive Structures of Choice of Higher Education”, British Journal of Sociology of Education, vol. 23, núm. 1, pp. 51-72.

Bernstein, Basil (1985), “Clases sociales, lenguaje y socialización”, Revista Colombiana de Educación, núm. VI, pp. 25-43.

Bogdan, Sojkin, Paweł Bartkowiak y Agnieszka Skuza (2012), "Determinants of Higher Education Choices and Student Satisfaction: the Case of Poland", Higher Education, vol. 63, núm. 5, pp. 565-581.

Boudon, Raymond (1973), Education, Opportunity, and Social Inequality; Changing Prospects in Western Society, Nueva York, Wiley.

Bourdieu, Pierre y Jean Claude Passeron (1996) [1977], La reproducción. Elementos para una teoría de la enseñanza, segunda edición, México, Fontarama. 
Breen, Richard y John Goldthorpe (1997), "Explaining Educational Differentials: towards a Formal Rational Action Theory", Rational and Society, núm. 9, pp. 275-305.

Büchner, Charlotte y Rolf Van del Velden (2013), "How Social Background Affects Educational Attainment over Time in the Netherlands", en M. Jackson (ed.), Determined to Succeed? Performance versus Choice in Educational Attainment, Stanford, Stanford University Press.

Chen, Liang-Hsuan (2007), "Choosing Canadian Graduate Schools from Afar: East Asian Students' Perspectives”, Higher Education, núm. 54, pp. 759-780.

Contini, Dalit y Andrea Scagni (2013), "Social Origin Inequalities in Educational Careers in Italy. Performance or Decision Effects?", en M. Jackson (ed.), Determined to Succeed? Performance versus Choice in Educational Attainment, Stanford, Stanford University Press.

Cubillo, José María, Joaquín Sánchez y Julio Cerviño (2006), “International Students' Decision-Making Process", International Journal of Educational Management, vol. 20, núm. 2, pp. 101-115.

Davey, Gayna (2012), "Beyond a Binary Model of Students' Educational DecisionMaking”, Sociological Research Online, vol. 17, núm. 3, en URL http://www. socresonline.org.uk/17/3/4.html, fecha de consulta mayo de 2013.

Didou, Sylvie (2005), Internacionalización y proveedores externos de educación superior en América Latina y el Caribe, México, ANUIES.

Didou, Sylvie y Etienne Gérard (2011), "El Sistema Nacional de Investigadores en 2009. ¿Un vector para la internacionalización de las élites científicas?”, Perfiles Educativos, vol. XXXIII, núm. 132.

Elder, Glen H. Jr. (1992), “The Life Course”, en E. Borgatta y B. Marie (eds.), The Encyclopedia of Sociology, Nueva York, MacMillan, pp. 1120-1130.

Findlay, Allan, Russell King, Alexandra Stam y Enric Ruiz-Gelices (2006), "Ever Reluctant Europeans. The Changing Geographies of UK Students Studying and Working Abroad", European Urban and Regional Studies, vol. 13, núm. 4, pp. 291-318.

Galotti, Kathleen M., Elizabeth Ciner, Hope E. Altenbaumer, Heather J. Geerts, Allison Rupp y Julie Woulfe (2006), "Decision-Making Styles in a Real-Life Decision: Choosing a College Major", Personality and Individual Differences, núm. 41, pp. 629-639.

Gérard, Etienne (2014), "Las vías de la internacionalización de la ciencia, entre herencia y lógicas de competencia en el mercado de las formaciones de alto nivel", Conferencia en el contexto de las actividades del 40 aniversario de la UAM, 15 de abril, México.

Gérard, Etienne y Estela Maldonado (2009), “'Polos de saber' y 'cadenas de saber'. Impactos de la movilidad estudiantil en la estructuración del campo científico mexicano", Revista de la Educación Superior, vol. XXXVIII (4), núm. 152, octubre-diciembre, pp. 49-62.

Gérard, Etienne y Rocío Grediaga (2009), "Endogamia o exogamia científica. La formación en el extranjero, una fuerte influencia en las prácticas y redes científicas, 
en particular en las ciencias duras", Seminario Internacional Fuga de cerebros, movilidad académica y redes científicas, 2-4 de marzo, México, Cinvestav/IRD. González Barea, Eva María (2008), "Un proceso migratorio estudiantil (pre-migración, migración y post-migración): jóvenes marroquíes en la Universidad de Granada", Revista Electrónica de Investigación Educativa, vol. 10, núm. 2.

Grediaga, Rocío y Estela Maldonado (2012), "Polos de formación y políticas públicas. Un primer acercamiento a la reconstrucción de las trayectorias de formación de los científicos mexicanos", XI Congreso Nacional de Investigación Educativa, 7-11 de noviembre, Monterrey.

Greenbank, Paul (2009), "Re-Evaluating the Role of Social Capital in the Career Decision-Making Behavior of Working-Class Students", Research in PostCompulsory Education, vol. 14, núm. 2, pp. 157-170.

Heinz, Walter R. (2009), "Structure and Agency in Transition Research", Journal of Education and Work, vol. 22, núm. 5, pp. 391-404.

Hodkinson, Phil y Andrew C. Sparkes (1997), "Careership: a Sociological Theory of Career Decision Making", British Journal of Sociology of Education, vol. 18, núm. 1, pp. 29-44.

Jackson, Michelle (ed.) (2013), Determined to Succeed? Performance versus Choice in Educational Attainment, Stanford, Stanford University Press.

Luchilo, Lucas (2008), Evaluación de impacto del programa de formación de científicos y tecnólogos 1997-2006, México, Conacyt, Centro Redes.

Mei, Li y Mark Bray (2007), "Cross-Border Flows of Students for Higher Education: Push-Pull Factors and Motivations of Mainland Chinese Students in Hong Kong and Macau", Higher Education, núm. 53, pp. 791-818.

Neugebauer, Martin, David Reimer, Steffen Schindler y Volker Stocké (2013), "Inequality in Transitions to Secondary School and Tertiary Education in Germany", en M. Jackson (ed.), Determined to Succeed? Performance versus Choice in Educational Attainment, Stanford, Stanford University Press.

Reay, Diane, Miriam David y Stephen Ball (2001), "Making a Difference?: Institutional Habituses and Higher Education Choice", Sociological Research Online, vol. 5, núm. 4, en URL http://www.socresonline.org.uk/5/4/reay.html, fecha de consulta febrero de 2014.

Rehaag Tobey, Irmgard María (2008), "Una estancia de estudios en el extranjero. Experiencias en tres continentes", Revista de la Educación Superior, vol. XXXVII (3), núm. 147, julio-septiembre.

Salisbury, Mark, Paul Umbach, Michael Paulsen y Ernest Pascarella (2009), “Going Global: Understanding the Choice Process of the Intent to Study Abroad", Research in Higher Education, vol. 50, pp. 119-143.

Spitzberg, Brian H. (1997), "A Model of Intercultural Communication Competence", en L. Samovar, R. Porter y E. McDaniel, Intercultural Communication: a Reader, Wadsworth, Boston.

Tuirán, Rodolfo (2009), "Prefacio", en Sylvie Didou y Etienne Gérard (eds.), Fuga de cerebros, movilidad académica y redes científicas. Perspectivas latinoamericanas, México, IESALC, Cinvestav, IRD. 
UNESCO (Organización de las Nacionales Unidas para la Educación, la Ciencia y la Cultura) (2013), "Global Flow of Tertiary-Level Students", en URL http://www. uis.unesco.org/EDUCATION/Pages/international-student-flow-viz.aspx, fecha de consulta marzo de 2013.

Van Der Meid, J. Scott (2003), “Asian Americans: Factors Influencing the Decision to Study Abroad", Frontiers: the Interdisciplinary Journal of Study Abroad, vol. 9, otoño, pp. 71-110.

Van Mol, Christof (2008), "La migración de estudiantes chinos hacia Europa", Migraciones Internacionales, vol. 4, núm. 4, julio-diciembre.

Waters, Johanna y Rachel Brooks (2010), “Accidental Achievers? International Higher Education, Class Reproduction and Privilege in the Experiences of UK Students Overseas", British Journal of Sociology of Education, vol. 31, núm. 2, marzo. West, Anne, Apostolis Dimitropoulos, Audrey Hind y John Wilkes (2000), "Reasons for Studying Abroad: a Survey of EU Students Studying in the UK", European Conference on Education Research, 20-23 de septiembre, Edimburgo.

\section{Acerca de la autora}

Mónica López Ramírez es candidata a doctora en Ciencia Social con especialidad en Sociología por el Centro de Estudios Sociológicos de El Colegio de México. Sus áreas de interés son internacionalización de la educación superior, movilidad internacional de estudiantes y los procesos de socialización de estudiantes de posgrado. Dos obras recientes son "Desigualdades tardías: origen social y elección de destinos nacionales y extranjeros para estudiar el doctorado", en Alain Basail y Óscar F. Contreras (coords.), La construcción del futuro: los retos de las ciencias sociales en México. Memorias del 4to. Congreso Nacional de Ciencias Sociales, Tuxtla Gutiérrez y Tijuana, CESMECA-UNICACH y COMECSO, 2014, pp. 585-598; y en coautoría con Rocío Grediaga, "Los niveles de consolidación de los programas en estudio", en R. Grediaga (coord.), Socialización de la nueva generación de investigadores en México. Consolidación, recambio o renovación de la planta académica nacional, México, ANUIES, 2012. 